\section{Aggressive case of early onset gastric cancer; identifying the knowledge gap}

\section{INTRODUCTION}

A woman aged 30 years was referred for endoscopy for iron deficiency anaemia. Colonoscopy was normal. Upper endoscopy demonstrated multiple small, carpet-like, sessile polyps in the gastric fundus and a larger $4 \mathrm{~cm}$ polyp in the body (figure 1 ). The duodenum was normal without polyps. Endoscopic ultrasound demonstrated a $35 \times 36 \mathrm{~mm}$ hypoechoic polypoid lesion arising

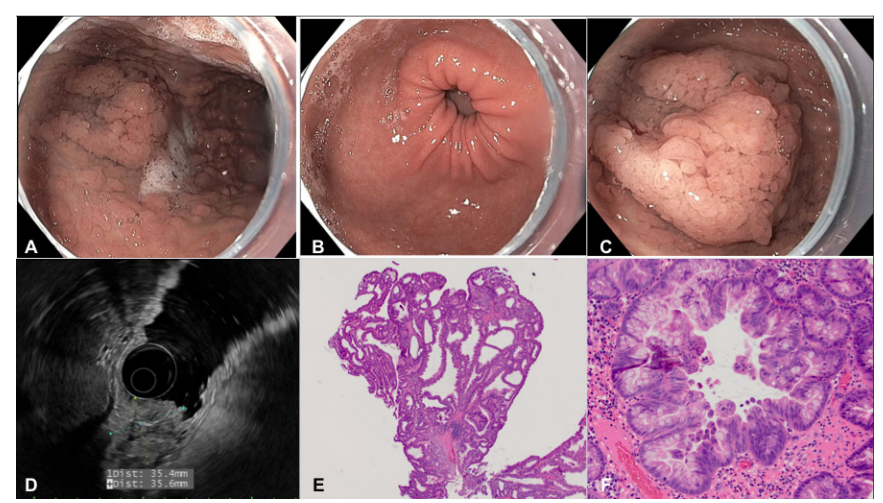

Figure 1 Upper endoscopy $(A-C)$ and endoscopic ultrasound (D), with corresponding pathology $(E, F)$. (A) Gastric body, long view demonstrated multiple small, sessile polyps. (B) Gastric antrum, normal in appearance. (C) Gastric body, $4 \mathrm{~cm}$ polyp. (D) A $35 \times 36 \mathrm{~mm}$ lesion originating in the mucosa with invasion into the submucosa. (E) Lowpower image from the polypectomy specimen shows large, cysticallydilated crypts lined by foveolar-type epithelium, characteristic of a hyperplastic polyp (H\&E stain, 20x magnification). (F) Higher power image from the polypectomy specimen shows a focus of high-grade dysplasia characterised by loss of nuclear polarity, micropapillary architecture and large, rounded nuclei (H\&E stain, 200× magnification).

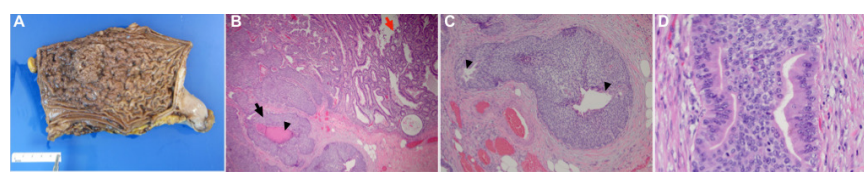

Figure 2 Gastrectomy specimen and corresponding pathology. (A) Gross photography from the gastrectomy specimen shows an altered appearance of rugal folds with innumerable polypoid lesions. (B) Lowpower image from the gastrectomy specimen shows a hyperplastic polyp (red arrow) overlying large nodules of malignant basaloid cells within the mucosa and submucosa (black arrow) with foci of central comedo-type necrosis (black arrowhead) (H\&E stain, 40x magnification). (C) Higher power image shows a nodule of malignant cells within the submucosa with associated desmoplastic stromal change. The nodule is predominantly comprised of basaloid cells and two foci of gland formation are seen (arrowhead), supporting that this lesion is an adenocarcinoma (H\&E stain, 100× magnification). (D) High-power image of the poorly differentiated adenocarcinoma demonstrates cells with mild nuclear pleomorphism and moderate pale to eosinophilic cytoplasm. Scattered mitotic figures are seen (H\&E stain, $400 \times$ magnification).

from the mucosa and invading into the submucosa, but with welldefined borders suggesting lack of invasion into adjacent structures. Endoscopic mucosal resection was performed on a portion of this polyp for histology. Pathology showed a focus of high-grade dysplasia arising in hyperplastic polyp. Her mother and maternal grandmother had gastric cancer, and her sister had gastric polyps and a non-melanoma skin cancer. The patient underwent a laparoscopic total gastrectomy and surgical pathology demonstrated a poorly differentiated adenocarcinoma with 23 negative lymph nodes, stage pT1bN0M0 (figure 2). No adjuvant therapy was recommended, and surveillance CT scan at 6 months was negative. Thirteen months after surgery, she presented with abdominal pain and CT scan showed a $3 \times 4.6 \mathrm{~cm}$ hypoattenuating lesion in the left hepatic lobe and a $<1 \mathrm{~cm}$ lesion in the right lobe. Biopsy demonstrated gastric adenocarcinoma consistent with metastatic disease. Systemic chemotherapy with 5-Fluorouracil, leucovorin, and oxaliplatin (FOLFOX) was initiated. Six months later, both liver lesions remain stable without other sites of disease recurrence.

\section{QUESTION}

What caused this young woman's aggressive gastric cancer?

See page 852 for answer 
Editor's quiz: Gl snapshot

\section{Aggressive case of early onset gastric cancer; identifying the knowledge gap}

See page 828 for question

\begin{abstract}
ANSWER
Genetic testing revealed a mutation in the $1 \mathrm{~B}$ promoter region of the APC gene (c.191T >C) consistent with gastric adenocarcinoma and proximal polyposis of the stomach (GAPPS). Hyperplastic and fundic gland polyps commonly occur in the stomach and are usually sporadic and non-neoplastic. However, GAPPS is an extremely rare syndrome with an autosomal dominant inheritance pattern characterised by extensive polyps carpeting the proximal stomach (fundus and body), often clustering with mass-like features, with antral/lesser curvature sparing and no duodenal/colon polyps or extraintestinal manifestations. ${ }^{1}$ These features distinguish GAPPS from sporadic benign polyps, familial adenomatous polyposis, MUTYH-associated polyposis, Peutz-Jeghers syndrome, juvenile polyposis syndrome and Cowden's syndrome. GAPPS was first reported in $2012^{2}$ and has since been identified in $<20$ families worldwide. ${ }^{34}$ There are no published recommendations on screening for family members, endoscopic surveillance, chemoprevention or timing of prophylactic gastrectomy. There are reported cases of individuals with polyps alone or with early cancer who later present with widespread metastases, similar to our patient, that support prophylactic gastrectomy. ${ }^{5}$ We share this case to draw attention to GAPPS and call for prospective registries to follow affected families and fill the knowledge gap with clinical guidelines.
\end{abstract}

\section{Jennifer M Kolb $\odot$,' Stephen Leong, ${ }^{2}$ Lindsey M Westbrook, ${ }^{3}$} Swati G Patel ${ }^{1}$

'Division of Gastroenterology \& Hepatology, University of Colorado Anschutz Medical Campus, Aurora, Colorado, USA

${ }^{2}$ Division of Medical Oncology, University of Colorado Anschutz Medical Campus, Aurora, Colorado, USA
${ }^{3}$ Department of Pathology, University of Colorado Anschutz Medical Campus, Aurora, Colorado, USA

Correspondence to Dr Jennifer M Kolb, Division of Gastroenterology \& Hepatology, University of Colorado - Anschutz Medical Campus, Aurora, CO 80045, USA; jennifer.m.kolb@cuanschutz.edu

Correction notice This article has been corrected since it published Online First. A typographical error has been corrected in the legend of figure 2 .

Twitter Jennifer M Kolb @jenkolbmd

Acknowledgements We thank Ms Allison Richards, Ms Amanda Gould and their family.

Contributors Drafting the manuscript: JK, critical revision: SL and SP.

Funding The authors have not declared a specific grant for this research from any funding agency in the public, commercial or not-for-profit sectors.

Competing interests None declared.

Patient consent for publication Obtained.

Provenance and peer review Not commissioned; externally peer reviewed.

(C) Author(s) (or their employer(s)) 2021. No commercial re-use. See rights and permissions. Published by BMJ.

D) Check for updates

To cite Kolb JM, Leong S, Westbrook LM, et al. Gut 2021;70:852.

Received 11 December 2019

Revised 10 January 2020

Accepted 13 January 2020

Published Online First 11 March 2020

Gut 2021;70:852. doi:10.1136/gutjnl-2019-320453

ORCID iD

Jennifer M Kolb http://orcid.org/0000-0002-6352-0797

\section{REFERENCES}

1 Beer A, Streubel B, Asari R, et al. Gastric adenocarcinoma and proximal polyposis of the stomach (GAPPS) - a rare recently described gastric polyposis syndrome - report of a case. Z Gastroenterol 2017;55:1131-4.

2 Worthley DL, Phillips KD, Wayte N, et al. Gastric adenocarcinoma and proximal polyposis of the stomach (GAPPS): a new autosomal dominant syndrome. Gut 2012:61:774-9.

3 Repak R, Kohoutova D, Podhola M, et al. The first European family with gastric adenocarcinoma and proximal polyposis of the stomach: case report and review of the literature. Gastrointest Endosc 2016;84:718-25.

4 Mitsui Y, Yokoyama R, Fujimoto S, et al. First report of an Asian family with gastric adenocarcinoma and proximal polyposis of the stomach (GAPPS) revealed with the germline mutation of the APC exon 1B promoter region. Gastric Cancer 2018;21:1058-63.

5 Rudloff U. Gastric adenocarcinoma and proximal polyposis of the stomach: diagnosis and clinical perspectives. Clin Exp Gastroenterol 2018;11:447-59. 